\title{
Impact on Soft Sand: Void Collapse and Jet Formation
}

\author{
Detlef Lohse, Raymond Bergmann, René Mikkelsen, Christiaan Zeilstra, Devaraj van der Meer, Michel Versluis, \\ Ko van der Weele, Martin van der Hoef, and Hans Kuipers
}

Faculty of Science and J. M. Burgers Centre for Fluid Dynamics, University of Twente, 7500 AE Enschede, The Netherlands (Received 1 May 2004; revised manuscript received 30 August 2004; published 3 November 2004)

\begin{abstract}
Very fine sand is prepared in a well-defined and fully decompactified state by letting gas bubble through it. After turning off the gas stream, a steel ball is dropped on the sand. On impact of the ball, sand is blown away in all directions ("splash") and an impact crater forms. When this cavity collapses, a granular jet emerges and is driven straight into the air. A second jet goes downwards into the air bubble entrained during the process, thus pushing surface material deep into the ground. The air bubble rises slowly towards the surface, causing a granular eruption. In addition to the experiments and the discrete particle simulations we present a simple continuum theory to account for the void collapse leading to the formation of the upward and downward jets.
\end{abstract}

DOI: 10.1103/PhysRevLett.93.198003

According to Shoemaker, the "impact of solid bodies is the most fundamental process that has taken place on the terrestrial planets" [1], as they shape the surfaces of all solar system bodies. A lot of information on this process has been extracted from remote observations of impact craters on planetary surfaces. However, the nature of the geophysical impact events is that they are nonreproducible. Moreover, their scale is enormous and direct observations are not possible. On the other hand, Thoroddsen and Shen did small scale experiments by letting a lead sphere fall on monodisperse spherical glass beads [2]. They found a jet emerging from the impact site. Similar jets are long known when a ball or a fluid droplet impacts on a liquid surface [3-7]. We did similar experiments as in [2], but now on extremely fine sand (average grain size of about $40 \mu \mathrm{m}$; grains are nonspherical) [8]. We found it hard to achieve quantitatively reproducible results, presumably due to the random nature of the force-chain networks in the granular material [9-12]. Therefore, in order to prepare a well-defined initial state, we decompactify and homogenize the fine sand by blowing air through it via a perforated bottom plate. The height of the sand bed above the bottom plate is typically 25$40 \mathrm{~cm}$. The air is slowly turned off before the experiments and the grains are left to settle in an extremely loose packing (41\% volume fraction) with the force chains either broken or substantially weakened. We call this a "fluidlike" state. Impact events on this well-prepared fine sand will be gravity dominated. We let a steel ball (radius $R_{0}=1.25 \mathrm{~cm}$ ) fall from various heights (up to $1.5 \mathrm{~m}$ ) onto the sand and observe the dynamics of the sand with a digital high-speed camera (up to 2000 frames per second).

The series of visible events is as follows (see Fig. 1): First, the ball vanishes in the sand and a crownlike splash is created. Inhomogeneities develop in the crown, due to the inelastic particle-particle interaction (Fig. 1, frames 3-5). Then, after a while, a jet shoots out of the sand at the position of impact. In all our experiments the
PACS numbers: 45.70.-n, 96.20.Ka, 96.35.Gt

jet height exceeds the release height of the ball; see Fig. 2(a). While the upper part of the jet is still going upwards, in the lower parts the inelastic particle-particle collisions lead to density inhomogeneities in the jet

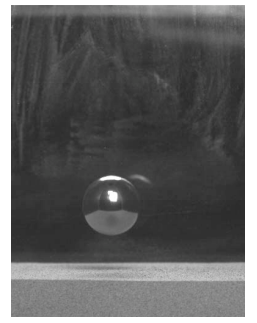

$t=-7 \mathrm{~ms}$

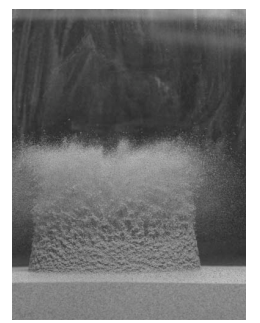

$t=88 \mathrm{~ms}$

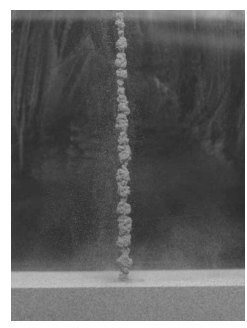

$t=358 \mathrm{~ms}$

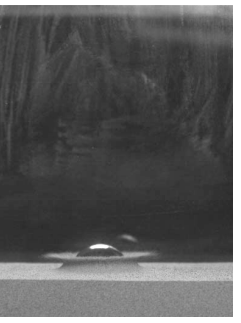

$t=5 \mathrm{~ms}$

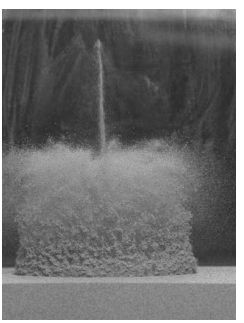

$t=108 \mathrm{~ms}$

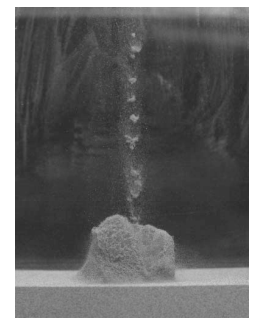

$t=503 \mathrm{~ms}$

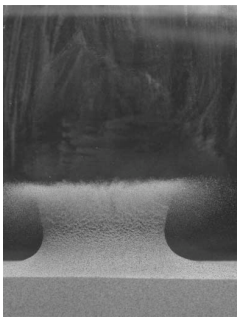

$t=33 \mathrm{~ms}$

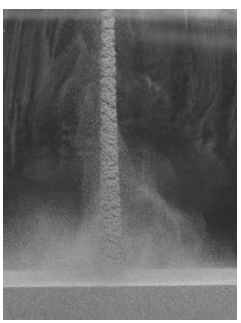

$t=233 \mathrm{~ms}$

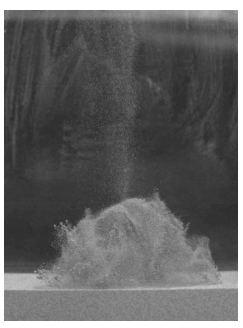

$t=583 \mathrm{~ms}$
FIG. 1. Jet formation after the impact $\left(v_{0}=2.43 \mathrm{~m} / \mathrm{s}\right)$ of a steel ball of $R_{0}=1.25 \mathrm{~cm}$ on loose very fine sand. The jet in this experiment exceeds the release height of the ball. Frames 2-4: splash; frames 5-6: a jet emerges; frame 7: clustering within the jet; frames 8-9: granular eruption at the surface. 

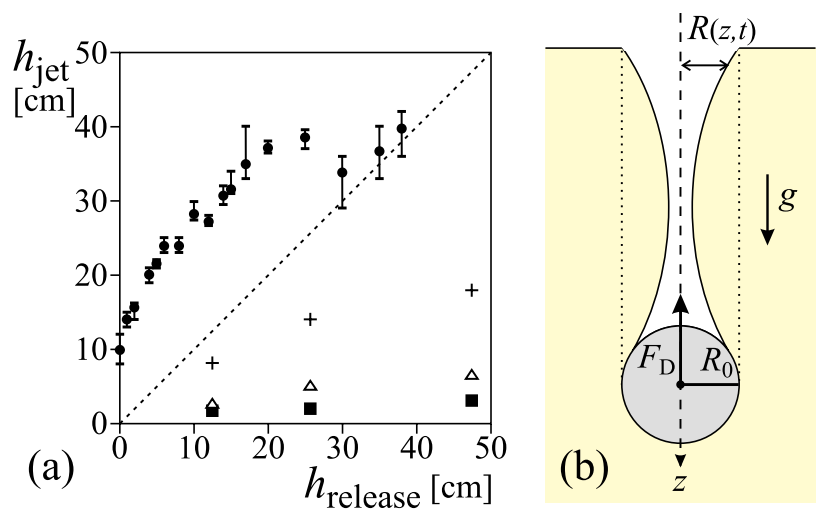

FIG. 2 (color online). (a) Jet height as a function of release height of the ball in our experiments (solid bullets with error bars) and in those of Ref. [2] [for spheres in a granulate of a different diameter: $d_{s}=0.08 \mathrm{~mm}$ (pluses), $d_{s}=0.118 \mathrm{~mm}$ (triangles), $d_{s}=0.176 \mathrm{~mm}$ (solid squares)]. The jets of Ref. [2] never reach the release height, because the granulate is less fine and much less decompactified. In our experiments jets are produced even at zero impact height [21] and there is no scaling relation as in Ref. [2]. (b) Sketch of the void collapse. When the accelerated sand grains from the sidewalls of the cylindrical cavity collide on the axis of the cavity, two jets are formed: one downward into the entrained air bubble formed above the sphere, and one upward straight into the air.

(Fig. 1, frames 7-8). These inhomogeneities resemble those of the surface tension driven Rayleigh instability of a water jet, even though there is no surface tension in granular matter. Finally, after about half a second, a granular eruption is seen at the position of impact, resembling a volcano (Fig. 1, frames 8-9). The collapsing jet first leaves a central peak in the crater [13-15], but the granular eruption violently erases this peak.

How does the jet form? To find out what is going on below the surface of the sand, we (i) performed direct numerical simulations, (ii) redid the experiments in two dimensions, meaning that we replaced the ball by a cylinder (with the axis parallel to the surface and orthogonal to the side plates) which we let fall into a bed of sand between two transparent plates, and (iii) employed the analogy to jet formation in fluids $[3-7,16]$.

(i) In the discrete particle simulations, the sand particles are modeled as spheres which interact via inelastic "soft-sphere" collision rules. The interaction of the particles with the surrounding air is included via empirical drag force relations [17]. Since the maximum number of particles that can be simulated is presently of the order of $10^{6}$, we can perform only quasi-two-dimensional simulations, where the thickness of the sand bed between the parallel plates is eight grains [18]. Altogether, the calculation includes $N=1.3 \times 10^{6}$ homogeneous beads of density $1000 \mathrm{~kg} / \mathrm{m}^{3}$ and diameter $500 \mu \mathrm{m}$ (i.e., approximately a factor of 10 larger than in experiment) in a container of $24 \times 0.4 \mathrm{~cm}^{2}$ ground area and a sand bed height of about $17 \mathrm{~cm}$. The beads are prefluidized with air, just as in the experiments, and then a $1.5 \mathrm{~cm}$ diameter ball of density $3500 \mathrm{~kg} / \mathrm{m}^{3}$ is dropped onto the beads with an impact velocity of $2 \mathrm{~m} / \mathrm{s}$. The series of events can be seen in Fig. 3, revealing the jet formation process invisible in Fig. 1: The impacting ball creates a void which is then pressed together through the "hydrostatic" pressure from the side which nonlinearly increases with depth [19]. At small depth the ball passes early, meaning an early start of the void collapse, which however is weak due to the small hydrostatic pressure. Conversely, at larger depth the collapse of the void begins later but is stronger due to the larger hydrostatic pressure. Somewhere in the middle the collapse is finished first, and the void walls hit each other. It is this singularity which leads to the formation of $t w o$ jets: One upwards and one downwards into an air bubble which was entrained in the sand by the void collapse. The falling jet often leaves a central peak in the crater (which in our 3D experiments with the fine, decompactified sand is subsequently erased again by the granular eruption). Note that the jet in the discrete particle simulations is much less pronounced than in experiment. First, because the beads in the simulations are much larger than the sand grains in the experiment; i.e., the sand bed is less fluidlike and allows for less fine structure. Second, the singularity due to the focusing along the axis of symmetry is weaker in $2 \mathrm{D}$ and quasi-2D experiments or simulations than in $3 \mathrm{D}$, and the jet takes the form of a sheet. We will present a quantitative comparison of experimental and numerical impact events on $500 \mu \mathrm{m}$ beads elsewhere.

(ii) We performed such 2D jet formation experiments by letting a cylinder fall into decompactified sand between two transparent plates and observing the jet formation process from the side [8]. These experiments

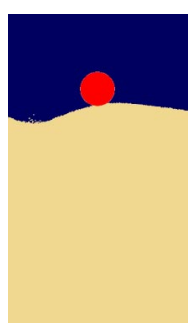

$t=0 \mathrm{~ms}$

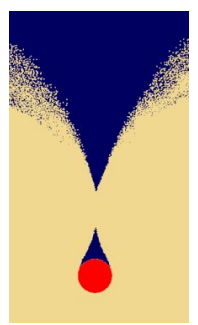

$t=245 \mathrm{~ms}$
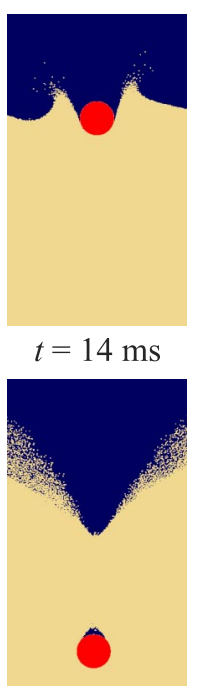

$t=273 \mathrm{~ms}$

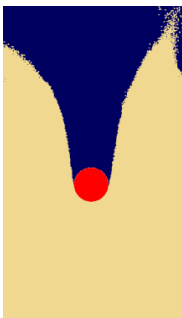

$t=69 \mathrm{~ms}$

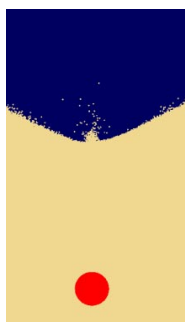

$t=385 \mathrm{~ms}$

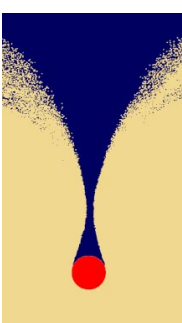

$t=224 \mathrm{~ms}$

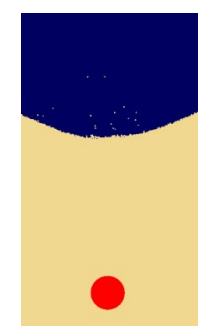

$t=495 \mathrm{~ms}$
FIG. 3 (color online). A cut through the quasi-2D discrete particle simulation. Frames 1-3: the impact of the disk on the particles; frames 4-6: the collapse of the void; frame 7: the upward jet (which is less pronounced than in the 3D experiments). 
confirm the above sketched series of events. Again, the jet is less pronounced than in the $3 \mathrm{D}$ experiments. The entrained air bubble slowly rises in these experiments, finally leading to a granular eruption at the surface, just as observed in $3 \mathrm{D}$.

(iii) The same series of events is also found after an analogous impact of a steel ball or a falling disk on water $[3-7,16,20]$. We employ this analogy in order to set up a "minimal" continuum mechanical model, in order to work out the essentials of the void collapse.

First, the delay curve $z(t)$ of the ball in the sand can be obtained from a simple force balance model involving drag, gravity, and added mass. It describes the experimental results obtained for a falling ball equipped with a thin stiff tail, which allows for easy depth measurements [21]. The delay curve $z(t)$ of the ball is inverted to obtain $t_{\text {pass }}(z)$, the time when the ball passes the layer of sand at depth $z$. This sets the initial conditions for the twodimensional collapse of the void at this depth, namely, $R\left(z, t_{\text {pass }}\right)=R_{0}$ and $\dot{R}\left(z, t_{\text {pass }}\right)=0$. Here, $R(z, t)$ is the time and depth dependent radius of the void; see Fig. 2(b).

Next, the collapse of the void formed by the ball has to be described. It is driven by the (hydrostatic) sand pressure $p(z)$ at depth $z$. For small $z$ the pressure simply is $p(z)=\rho_{s} g z$; for larger $z$ it saturates [19]. Here, $\rho_{s}$ is the sand density, assumed to be constant. If we neglect the dissipative processes both between the different layers of sand and between the sand grains in one layer, the dynamics for fixed depth $z$ is determined by the Euler equation,

$$
\rho_{s}\left[\partial_{t} v(r, t)+v(r, t) \partial_{r} v(r, t)\right]=-\partial_{r} p(r, t) .
$$

Here, $v(r, t)$ is the velocity field in the sand. With continuity $\partial_{r}[r v(r, t)]=0$, and with the boundary conditions $v(R(t), t)=\dot{R}(t)$ at the void's wall and $v\left(R_{\infty}, t\right)=0$ far away from the void, one obtains a Rayleigh-type [22,23] ordinary differential equation for each $R(z, t)$, namely,

$$
\left(R \ddot{R}+\dot{R}^{2}\right) \log \frac{R}{R_{\infty}}+\frac{1}{2} \dot{R}^{2}=\frac{1}{\rho_{s}} p(z)=g z .
$$

The radius $R_{\infty}$ is of the order of the system size, but the results only weakly (logarithmically) depend on this parameter. The dynamics following from this Rayleightype model is shown in Fig. 4, resembling the void collapse in the discrete particle simulations (Fig. 3), in the 2D experiments [8], in experimental work on the void collapse in transparent fluids $[3-7,16]$, in boundary integral simulations of the complete hydrodynamical equations [20], and therefore presumably also in the 3D experiments in sand shown in Fig. 1. Just before and at the singularity $[R(t)=0$ and diverging velocity], the dynamics is determined by $R \ddot{R}+\dot{R}^{2}=0$, which has the solution $R(t) \sim\left(t_{s}-t\right)^{1 / 2}$, where $t_{s}$ is the time of the singularity. The velocity therefore has a square-root divergence $\dot{R}(t) \sim\left(t_{s}-t\right)^{-1 / 2}$.
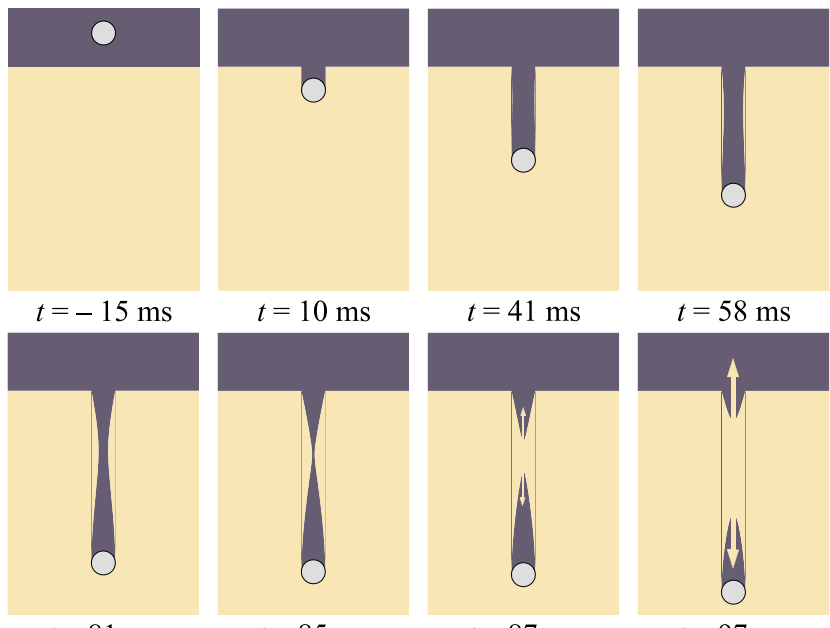

$t=81 \mathrm{~ms}$

$t=85 \mathrm{~ms}$

$t=87 \mathrm{~ms}$

$t=97 \mathrm{~ms}$

FIG. 4 (color online). A cross section of the 3D-void collapse following from our Rayleigh-type model, for the same impact velocity and ball radius as in Fig. 1. The void is pressed together by the hydrostatic pressure from the side, leading to a singularity and an upward and downward jet.

Having shown that the void collapse is driven by hydrostatic pressure, we now can deduce scaling arguments [24] for the limiting case of large impact velocity $v_{0}$, which is the relevant one in the geophysical context. The time up to void collapse in depth $z$ is the sum of the time $z / v_{0}$ it takes the ball to get there and the collapse time itself, which scales as $\sim R_{0} / \sqrt{g z}$. The depth $z_{c}$ where the walls of the void first touch (i.e., the position of the singularity) can be obtained from minimizing this sum with respect to $z$, resulting in $z_{c} / R_{0} \sim \mathrm{Fr}^{1 / 3}$, where $\mathrm{Fr}=$ $v_{0}^{2} /\left(g R_{0}\right)$ is the Froude number. From this one obtains that the time of the collapse $t_{c}$ scales as $t_{c} \sim\left(R_{0} / v_{0}\right) \mathrm{Fr}^{1 / 3} \sim$ $\sqrt{R_{0} / g} \mathrm{Fr}^{-1 / 6}$ [24]. For large $v_{0}$ these scaling laws are consistent both with our continuum model and with our discrete particle simulations.

We now discuss the role of the ambient air and the ambient pressure for the evolution of the jet: (i) For (3D) experiments with very high impact velocities we observed that after the splash the crown goes inwards rather than outwards, due to the pressure reduction behind the fast projectile (Bernoulli's law). The crown, in fact, can fully close and the jet then hits the closed crown, leading to an explosionlike collision which spreads material all over the place. (ii) In impact experiments on very fine and loose sand but with strongly reduced ambient pressure the jet, in fact, is less pronounced than with ambient pressure [25]. For larger particles $(500 \mu \mathrm{m})$ we redid above discrete particle simulations, but now reduced the air pressure to zero (vacuum) after fluidization. This gives very similar results for the jet formation. More research on the role of air in loose fine granular material clearly is required.

We conclude the Letter with speculations on possible implications of our findings on the impact mechanism 
within the geophysical context [14,26-30]. In general, impacts can be described using two dimensionless parameters: the Froude number $\mathrm{Fr}=V^{2} / g R$ and the (inverse) Newton number $\mathrm{Nt}^{-1}=\rho V^{2} / Y$, where $\rho, V$, and $R$ are the density, velocity, and radius of the impactor, respectively, and $Y$ is the yield stress of the target [30]. Although the absolute energy scales in our experiment are of course very different as compared to geophysical events, the relative scales are comparable: very largescale planetary impacts $(Y \approx 10 \mathrm{kbar}, R \approx 100 \mathrm{~km}$, and $V \approx 10 \mathrm{~km} / \mathrm{s})$ and our experiments $(Y \approx 10 \mathrm{~Pa}, R \approx$ $1 \mathrm{~cm}$, and $V \approx 3 \mathrm{~m} / \mathrm{s}$ ) both lead to Froude and Newton numbers of the same order: $\mathrm{Fr} \approx 10^{2}$ and $\mathrm{Nt}^{-1} \approx 10^{3}$.

At this point we caution the reader to directly translate or upscale the results of our laboratory experiments to the geophysical scales as, in addition to the aforementioned difference in absolute energy scales, lack of reproducibility of the details typify geophysical events. Nonetheless, we believe that the following speculations may stimulate discussions in a geophysical context: (i) After the impact of a solid body on a planet, it may be the upward jet and not the splash which is the dominant source of planetary material transferred into space [28]. Similarly, an oblique jet resulting from an oblique impact (not shown here; in that case the jet goes backwards) allows for an enhanced backwards transport of material, as compared to the splash. (ii) The collapsing jet may contribute to the central peak often found in impact craters $[14,15]$. (iii) The downward jet will considerably change the layering of the sediments underneath a crater, as it provides a mechanism how surface material can be transported deep into the ground. In addition, a granular eruption will rearrange the sediment. Our suggested mechanism may shed new light on the sediment layering data found underneath the Chicxulub crater, which is a source of major controversy [31-34].

We thank H. J. Melosh and J. Smit for discussions. The work is part of the research program of FOM, which is financially supported by NWO, and R. B., R. M., C.Z., D. v. d. M., and M.V. acknowledge financial support.

[1] E. M. Shoemaker, in Impact and Explosion Cratering, edited by D. J. Roddy, R. O. Pepin, and R. B. Meril (Pergamon Press, New York, 1977), pp. 1-10.

[2] S.T. Thoroddsen and A. Q. Shen, Phys. Fluids 13, 4 (2001).

[3] A. M. Worthington, A Study of Splashes (Longman and Green, London, 1908).

[4] H. Oguz and A. Prosperetti, J. Fluid Mech. 219, 143 (1990).

[5] A. Prosperetti and H. Oguz, Annu. Rev. Fluid Mech. 25, 577 (1993).
[6] J. E. Hogrefe, N. L. Peffley, C. L. Goodridge, W. T. Shi, H. G. E. Hentschel, and D. P. Lathrop, Physica (Amsterdam) 123D, 183 (1998).

[7] D. Lohse, Phys. Today 56, No. 2, 36 (2003).

[8] R. Mikkelsen, M. Versluis, E. Koene, G.-W. Bruggert, D. van der Meer, K. van der Weele, and D. Lohse, Phys. Fluids 14, S14 (2002).

[9] C. H. Liu, S. R. Nagel, D. A. Schecter, S. N. Coppersmith, S. Majumdar, O. Narayan, and T. A. Witten, Science 269, 513 (1995).

[10] H. M. Jaeger, S. R. Nagel, and R. P. Behringer, Rev. Mod. Phys. 68, 1259 (1996)

[11] L. Kadanoff, Rev. Mod. Phys. 71, 435 (1999).

[12] C. S. O'Hern, S. A. Langer, A. J. Liu, and S. R. Nagel, Phys. Rev. Lett. 86, 111 (2001).

[13] Similar peaks are observed in many craters of the terrestrial planets $[14,15]$.

[14] P. H. Schultz, J. Geophys. Res. E 97, 16183 (1992).

[15] A. M. Walsh, K. E. Holloway, P. Habdas, and J. R. de Bruyn, Phys. Rev. Lett. 91, 104301 (2003).

[16] J.W. Glasheen and T. A. McMahon, Nature (London) 380, 340 (1996).

[17] J. Li and J. A. M. Kuipers, Chem. Eng. Sci. 58, 711 (2003).

[18] The 3D simulations we did are too strongly affected by finite size effects. Nevertheless, also for these simulations a jet emerges.

[19] J. Duran, Sands, Powders, and Grains (Springer, New York, 1999), 1st ed.

[20] S. Gaudet, Phys. Fluids 10, 2489 (1998).

[21] D. Lohse, R. Rauhé, R. Bergmann, and D. van der Meer, "Dry Quicksand" (to be published).

[22] L. Rayleigh, Philos. Mag. 34, 94 (1917).

[23] H. N. Oguz and A. Prosperetti, J. Fluid Mech. 257, 111 (1993).

[24] H. N. Oguz, A. Prosperetti, and A. R. Kolaini, J. Fluid Mech. 294, 181 (1995).

[25] Heinrich Jaeger (private communication).

[26] E. Pierazzo and H. J. Melosh, Annu. Rev. Earth Planet Sci. 28, 141 (2000).

[27] Impact and Explosion Cratering, edited by D. J. Roddy, R. O. Pepin, and R. B. Meril (Pergamon Press, New York, 1978).

[28] J. D. O'Keefe and T. J. Ahrens, Science 198, 1249 (1977); 234, 346 (1986).

[29] H. J. Melosh, Impact Cratering: A Geologic Process (Oxford University Press, Oxford, 1989).

[30] K. Holsapple, Annu. Rev. Earth Planet Sci. 21, 333 (1993).

[31] G. Keller, J. G. LopezOliva, W. Stinnesbeck, and T. Adatte, Geol. Soc. Am. Bull. 109, 410 (1997).

[32] J. Smit, Annu. Rev. Earth Planet Sci. 27, 75 (1999).

[33] L. E. Marin, V. L. Sharpton, J. U. Fucugauchi, J. Smit, P. Sikora, C. Carney, and M. Rebolledo-Vieyra, Int. Geol. Rev. 43, 1145 (2001).

[34] G. Keller, W. Stinnesbeck, T. Adatte, B. Holland, D. Stuben, M. Harting, C. De Leon, and J. De la Cruz, J. Geol. Soc. 160, 783 (2003). 